\title{
Reversible alternation between bipolar and unipolar resistive switching in polycrystalline barium strontium titanate thin films
}

Wan Shen, Regina Dittmann, and Rainer Waser

Citation: Journal of Applied Physics 107, 094506 (2010);

View online: https://doi.org/10.1063/1.3369285

View Table of Contents: http://aip.scitation.org/toc/jap/107/9

Published by the American Institute of Physics

\section{Articles you may be interested in}

Improved endurance behavior of resistive switching in $(\mathrm{Ba}, \mathrm{Sr}) \mathrm{TiO}_{3}$ thin films with $\mathrm{W}$ top electrode Applied Physics Letters 93, 222102 (2008); 10.1063/1.3039809

Resistive switching mechanism of $\mathrm{TiO}_{2}$ thin films grown by atomic-layer deposition Journal of Applied Physics 98, 033715 (2005); 10.1063/1.2001146

Conduction mechanism of $\mathrm{TiN} / \mathrm{HfO}{ }_{\mathrm{X}} / \mathrm{Pt}$ resistive switching memory: A trap-assisted-tunneling model Applied Physics Letters 99, 063507 (2011); 10.1063/1.3624472

The effect of oxygen vacancy on switching mechanism of $\mathrm{ZnO}$ resistive switching memory Applied Physics Letters 110, 073501 (2017); 10.1063/1.4976512

Unipolar resistive switching characteristics of $\mathrm{ZnO}$ thin films for nonvolatile memory applications Applied Physics Letters 92, 022110 (2008); 10.1063/1.2834852

Resistive switching and data reliability of epitaxial $(\mathrm{Ba}, \mathrm{Sr}) \mathrm{Ti}_{3}$ thin films Applied Physics Letters 88, 042901 (2006); 10.1063/1.2162860

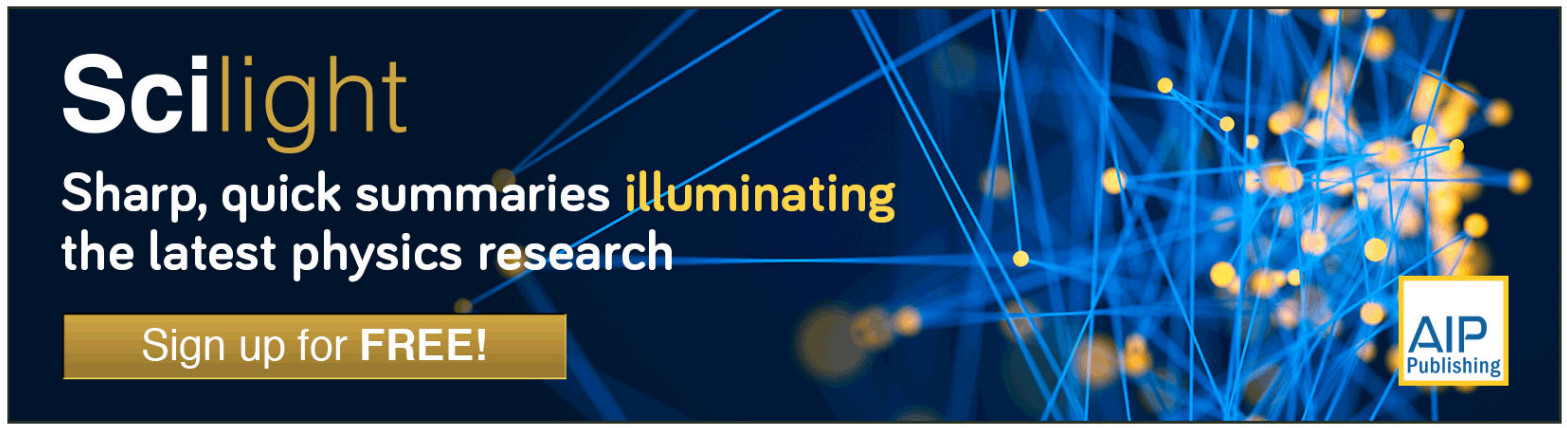




\title{
Reversible alternation between bipolar and unipolar resistive switching in polycrystalline barium strontium titanate thin films
}

\author{
Wan Shen, ${ }^{1, a)}$ Regina Dittmann, ${ }^{1}$ and Rainer Waser ${ }^{1}$ \\ ${ }^{1}$ Institute of Solid State Research, Research Center Juelich, 52425 Juelich, Germany and Juelich-Aachen \\ Research Alliance, Section Future Information Technologies (JARA-FIT), 52425 Juelich, Germany
}

(Received 11 January 2010; accepted 19 February 2010; published online 7 May 2010)

The alternation from bipolar to unipolar resistive switching was observed in perovskite $\mathrm{Ba}_{0.7} \mathrm{Sr}_{0.3} \mathrm{TiO}_{3}$ thin films. By controlling the switching voltage, either bipolar or unipolar switching was obtained. When the switching voltage is higher than a threshold voltage, the device exhibits unipolar switching while if the switching voltage is lower than a threshold voltage, the device shows bipolar switching behavior. The bipolar-to-unipolar alternation is dynamically repeatable and may be related to the local modification of broken filaments by oxygen vacancy movement. (c) 2010 American Institute of Physics. [doi:10.1063/1.3369285]

\section{INTRODUCTION}

The resistance reversibly changed by external electric stimulus has attracted a great deal of attention for the application as resistive random access memory (RRAM). The advantages such as low power consumption, high speed operation, nonreadout disturbance, and the high density integration potential made RRAM one of the most promising candidates for the next generation nonvolatile memory. ${ }^{1,2}$ There exist two schemes related to the electrical polarity required for the resistive switching. When the resistance change does not depend on the polarity of the voltage or current and the device can be switched between high resistance state (HRS) and low resistance state (LRS) with the same polarity, this polarity independent switching behavior is called unipolar switching. The second scheme is called bipolar switching because opposite polarity of the applied voltage is needed to switch between the HRS and LRS.

Unipolar switching and bipolar switching have been widely observed in simple binary metal oxide such as $\mathrm{NiO},{ }^{3}$ $\mathrm{Cu}_{2} \mathrm{O},{ }^{4} \mathrm{ZrO}_{2},{ }^{5}$ and $\mathrm{TiO}_{2},{ }^{6}$ while generally only the bipolar switching is observed for complex perovskite type oxide $\left(\mathrm{ABO}_{3}\right)$ films, such as $\mathrm{PCMO},{ }^{7} \mathrm{Cr}$-doped $\mathrm{SrZrO}_{3},{ }^{8}$ and $\left(\mathrm{Ba}_{0.7} \mathrm{Sr}_{0.3}\right) \mathrm{TiO}_{3}{ }^{9}$ The larger $\mathrm{R}_{\text {off }} / \mathrm{R}_{\text {on }}$ of unipolar switching oxides makes it much more easy to read the memory state ${ }^{10}$ and can be potentially integrated in higher density, ${ }^{11}$ while generally the bipolar switching has a better endurance.

Hence it would be advantageous to have both bipolar and unipolar switching schemes in the same material. For $\mathrm{TiO}_{2}$ thin films, a permanent transition from bipolar to unipolar has been observed. ${ }^{12}$ However, no reversible switching between the two switching schemes has been reported so far.

In this article, we report on the reproducible transformation between bipolar and unipolar resistive switching obtained in polycrystalline $0.2 \% \mathrm{Mn}$ doped $\mathrm{Ba}_{0.7} \mathrm{Sr}_{0.3} \mathrm{TiO}_{3}$ (BST) perovskite thin films with Pt bottom electrode.

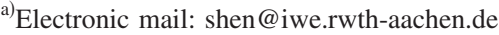

\section{EXPERIMENTAL}

The $200 \mathrm{~nm}$ thick $0.2 \% \mathrm{Mn}$ doped BST thin film was deposited on a $\mathrm{Pt} / \mathrm{Ti} / \mathrm{SiO}_{2} / \mathrm{Si}$ substrate at $700{ }^{\circ} \mathrm{C}$ by pulsed laser deposition at an oxygen pressure of 0.25 mbar. The BST film is grown in polycrystalline form confirmed by $\mathrm{x}$-ray diffraction measurements.

To achieve Pt/BST/Pt cells, $100 \mathrm{~nm}$ thick Pt was sputtered and subsequently the top electrodes were patterned by lithography and dry etching to areas of $0.04 \mathrm{~mm}^{2}$. The current-voltage (I-V) characteristics were measured using Agilent semiconductor analyzer B1500A. For electrical characterization, the voltage was applied to the top electrode and the bottom electrode was grounded. The I-V characteristics were started at $0 \mathrm{~V}$. The sweeping directions are indicated by arrows in the I-V characteristics. All measurements were performed at room temperature.

\section{RESULTS AND DISCUSSION}

The as-prepared Pt/BST/Pt devices were highly insulating and no resistive switching behavior is observed. Thus a forming process is necessary to trigger the device from the initial highly insulating state to a more conductive state as demonstrated in Fig. 1(a). The forming voltage is $20 \mathrm{~V}$ with a current compliance of $1 \mathrm{~mA}$ to protect the device from a permanent breakdown. After the first forming process, the device shows weak bipolar switching as illustrated in Fig. 1(b). The switching polarity of the I-V curve is identical with the former reported for epitaxial BST thin films, namely clockwise in the positive voltage branch and counterclockwise in the negative voltage branch. However, the $\mathrm{R}_{\text {off }} / \mathrm{R}_{\text {on }}$ is much smaller compared to our epitaxial BST thin film devices. ${ }^{9}$

If the current compliance is increased to $20 \mathrm{~mA}$, another forming occurs at positive branch (not shown here) and the device is transformed to a better conducting and linear behavior, as can be seen in Fig. 1(c), this linear branch acts as LRS of a unipolar switching state. When the voltage is swept to $1 \mathrm{~V}$ without a current compliance, the current dropped abruptly which means that the device was switched from the 

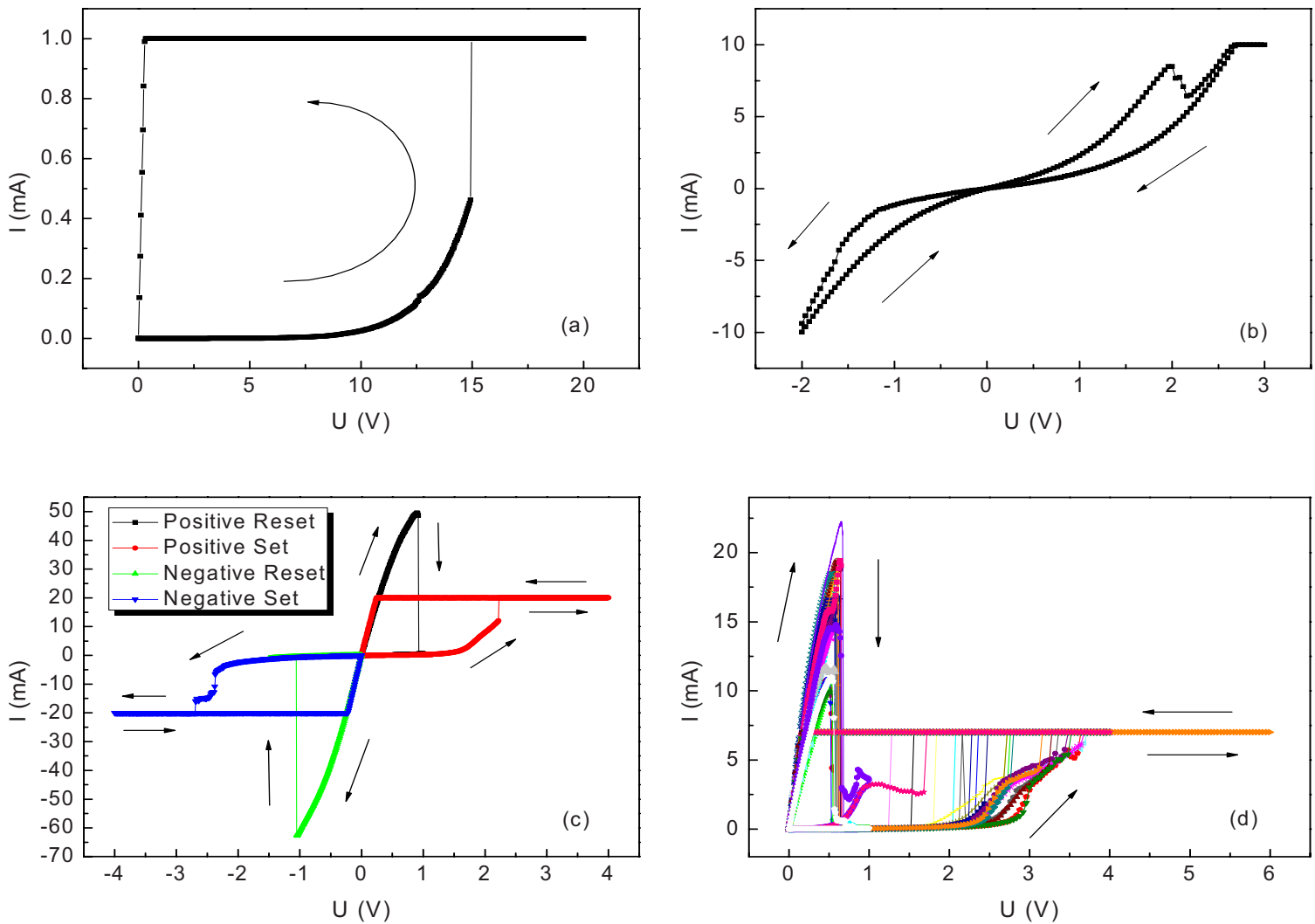

FIG. 1. (Color online) Resistive switching behavior of Pt/BST/Pt devices. (a) Forming process. (b) Bipolar switching behavior. (c) Unipolar switching behavior. (d) A Pt/BST/Pt device switched for 20 cycles in unipolar switching mode. The different set processes happened during the endurance measurement. The gradual or abrupt increase in resistance in set took place randomly, indicates the instability of HRS.

unipolar LRS to the unipolar HRS (reset process). While further increasing the voltage to $4 \mathrm{~V}$ with a current compliance of $20 \mathrm{~mA}$, the current jumps at about $2 \mathrm{~V}$ and the devices is switched to LRS again (set process). Set and reset process occurs symmetrically at the negative voltage branch. The $R_{\text {off }} / R_{\text {on }}$ ratio in the unipolar switching mode is more than 1000 and thereby much larger than the $R_{\text {off }} / R_{\text {on }}$ ratio in the bipolar switching mode shown in Fig. 1(b).

Figure 1(d) displays a Pt/BST/Pt device switched for 20 cycles in unipolar switching mode. It is clear that the different set processes happened during the endurance measurement. The gradual or abrupt decrease in resistance in set took place randomly, indicates the instability of HRS. ${ }^{13}$

Figure 2 shows the temperature dependence of both LRS

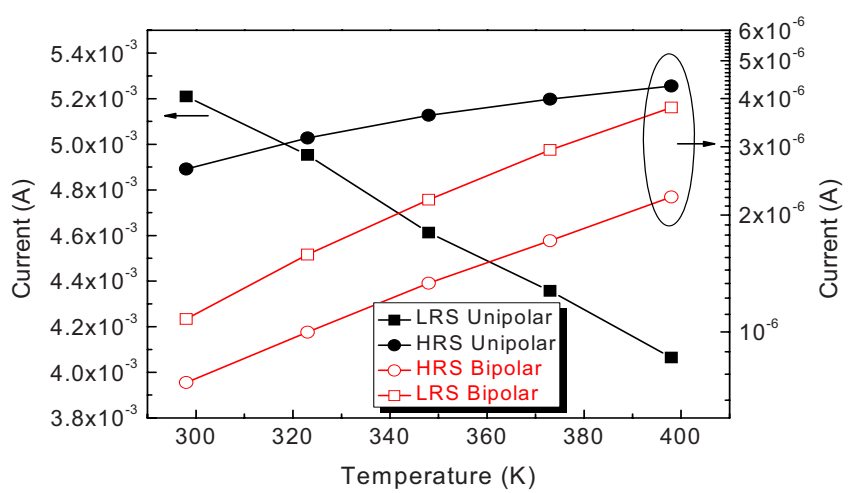

FIG. 2. (Color online) The temperature dependence of both bipolar and unipolar switching. and HRS in bipolar and the unipolar switching mode. The transition from bipolar switching to unipolar switching induced by forming is accompanied by a change of the charge carrier transport in LRS, from the thermally activated transport to a metallic transport, while in HRS both the bipolar and unipolar switching mode show thermally activated charge carrier transport, as reported elsewhere. ${ }^{12}$

In general, bipolar switching in perovskite insulators is attributed to a voltage-driven oxygen vacancy migration ${ }^{14}$ within extended defects and the resulting redox-process related metal-to-insulator transition. While thermal effects generally are considered to play a minor role for bipolar switching, Joule heating plays a key role for the formation and rupture of metallic filaments in the unipolar switching mode. Chae et al. ${ }^{10}$ observed filaments in unipolar switching in $\mathrm{TiO}_{2}$ thin films and demonstrated that the local temperature of the filaments can reach $700-800 \mathrm{~K}$ by localized Joule heating. ${ }^{15}$ In case of unipolar switching, the local metal-toinsulator transition is driven by the energetically favored low valence state in the high-temperature region rather than by a voltage-induced accumulation of oxygen vacancies. ${ }^{16}$

Since a forming procedure which transforms insulating samples to a metallic LRS is prerequisite for the observation of unipolar switching, we have to regard the forming procedure in our case. After the first forming [Fig. 1(a)], semiconducting filaments are formed as proved by the temperature dependence of the LRS shown in Fig. 2. We conclude from this observation, that the creation of oxygen vacancies during the first forming procedure is insufficient for the formation a 

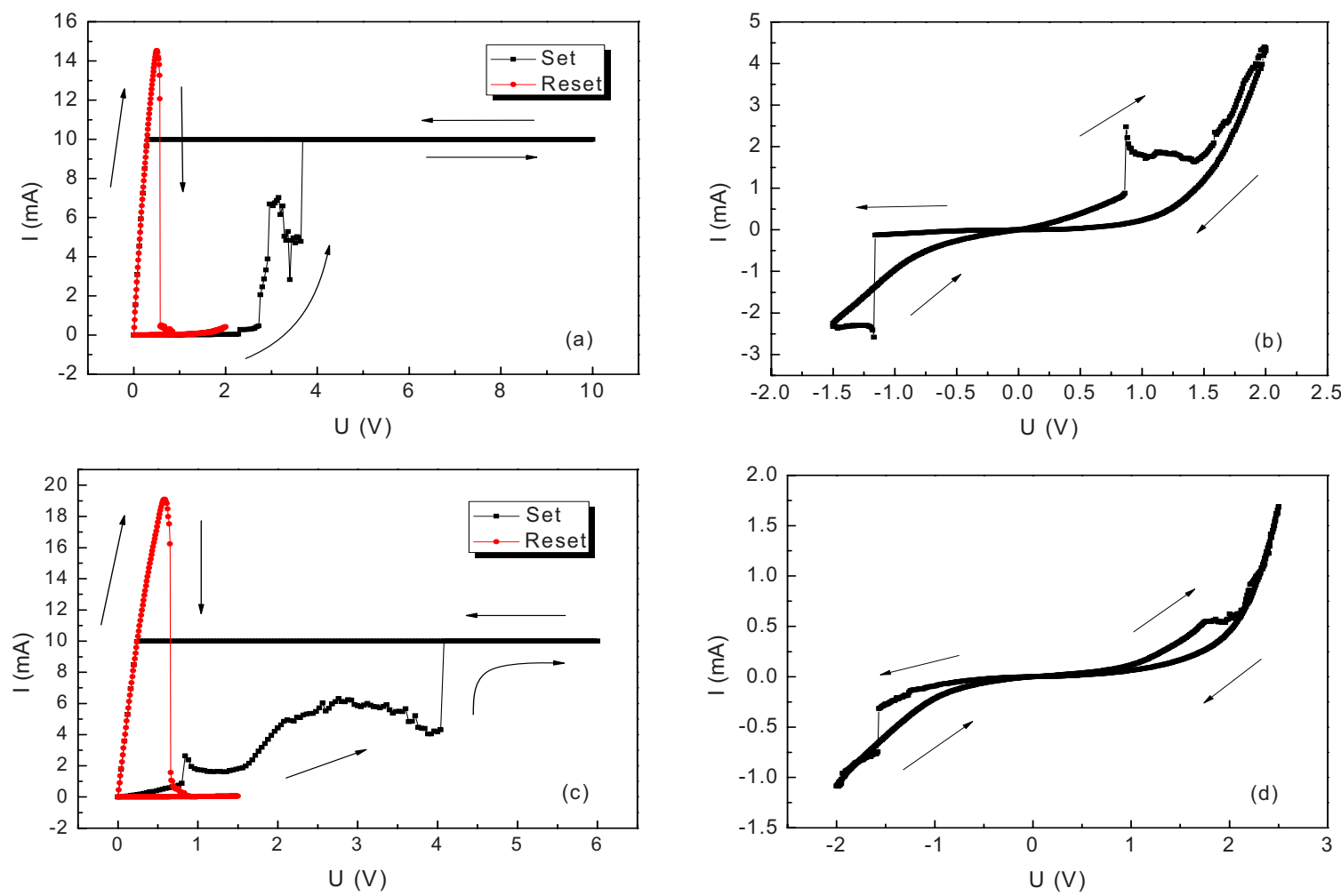

FIG. 3. (Color online) The unipolar/bipolar switching alternation by control of switching voltage. (a) Unipolar switching behavior when switching voltage is higher than threshold voltage $(\sim 4 \mathrm{~V})$. (b) Bipolar switching behavior when switching voltage is lower than threshold voltage. [(c) and (d)] Dynamically repeated unipolar/bipolar switching.

metallic filament. Once the LRS branch of the I-V curve has changed to a linear shape after the 2nd forming process [Fig. $1(\mathrm{c})]$, the filament property changed to metallic conduction behavior and the device exhibits unipolar switching.

Because the current compliance of $20 \mathrm{~mA}$ used in the 2nd forming procedure is higher than usually used for bipolar switching, a higher electrical power is applied to the sample and the dissipation of heat might become significant within the conducting filament. As a result, additional oxygen might be driven out of the filament due to the tendency of Ti to adopt a lower valence state at higher temperatures which can be identified with the unipolar LRS. The reset to the unipolar HRS process occurs, as soon as the threshold voltage for the thermal dissolution of the filaments is exceeded.

It should be noted that, if the current compliance used in the 1st forming is sufficient to completely reach the metallic LRS, the device will directly show unipolar switching. Accordingly, the forming current is of great importance and the metallic LRS is the prerequisite for the observation of unipolar switching in polycrystalline BST thin films.

Since we observed only bipolar switching in epitaxial BST films as reported before ${ }^{9}$ and no unipolar switching in epitaxial perovskite systems have been observed so far, grain boundaries have to play a crucial role for the realization of the unipolar switching mode. The importance of grain boundaries in terms of unipolar switching was already demonstrated by Park et al. ${ }^{17}$ Lee et $a l .{ }^{18}$ also observed that the epitaxial binary oxide $\mathrm{NiO}$ shows bipolar switching while the polycrystalline $\mathrm{NiO}$ shows unipolar switching.

The unipolar state obtained for our polycrystalline samples can be transformed back to a bipolar state as illustrated in Figs. 3(a)-3(d). In Fig. 3(a), the resistance of the HRS decreased gradually and the device was switched to the LRS at about $4 \mathrm{~V}$. When the sweeping voltage is chosen to 2 $\mathrm{V}$, which is below the threshold voltage of $\sim 4 \mathrm{~V}$ for the unipolar transition, the device is switched to an even higher HRS of a bipolar transition state, as shown in Fig. 3(b). As a result, the HRS in unipolar switching works as the LRS in bipolar switching mode. Therefore, we could convert the unipolar switching to bipolar switching by controlling the voltage, which should be smaller than the threshold voltage to avoid the soft breakdown.

However, as shown in Fig. 3(c), if we increase the voltage again beyond the threshold voltage, the resistance of the LRS in the bipolar switching mode was decreased further to a metallic LRS and the LRS of the unipolar switching was obtained. Moreover, when the sweeping voltage is decreased below the threshold voltage, the unipolar switching can be converted to bipolar switching again [Fig. 3(d)]. Consequently, we could obtain either bipolar switching or unipolar switching by controlling the switching voltage. This bipolar to unipolar transition is dynamically repeatable.

A simplified single filament model for the bipolar/ unipolar alternation is illustrated in Fig. 4. After unipolar set process, there will be a conductive channel (filament) formed in between the top and bottom electrodes, and in the reset process, the filament will not be completely but locally ruptured. Thus, in the following I-V sweep in bipolar switching mode, the oxygen movement results only in a conductance change at the spot of the broken filament. In this case the remaining filament will work as inner electrodes, or so called 

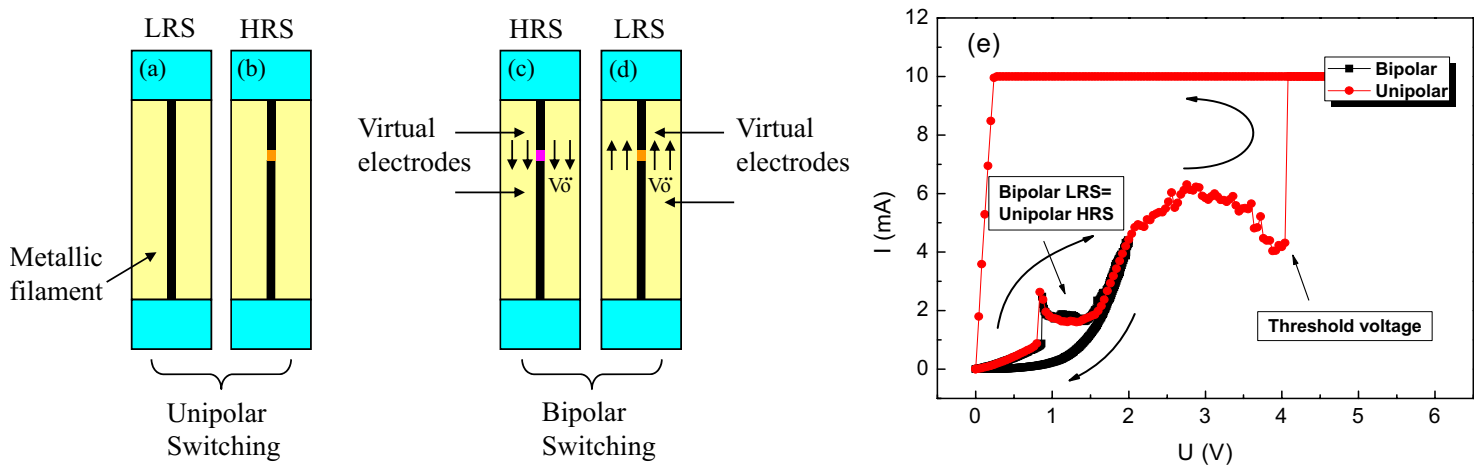

FIG. 4. (Color online) A simplified single filament model for unipolar/bipolar switching alternation. (a) The Pt/BST/Pt device shows unipolar LRS when metallic filament is formed and (b) device shows unipolar HRS when metallic filament is broken due to localized Joule heating effect. [(c) and (d)] The device shows bipolar switching when switching voltage is lower than threshold voltage. Oxygen vacancies could migrate through the trace of broken filament and the conductance of the device was locally modified. (e) The merged positive branch of Figs. 3(b) and 3(c).

"virtual electrode." During the second forming process the Ti within the filament is completely reduced to a metallic conductive phase within the filament. ${ }^{16}$ This state can be identified with the LRS of the unipolar switching mode depicted in Fig. 4(a). In the unipolar HRS [Fig. 4(b)], the filament is thermally dissolved due to local Joule heating.

Forming is an irreversible process and will result in a permanent change of the local composition of the virgin sample. However, the trace of the fused filament may still provide fast diffusion channels for oxygen vacancies compared to the highly insulating bulk material and the oxygen vacancy movement may locally modify the conductance of the fused filament. In such a case, the oxygen vacancies could migrate through the fused filament if a positive bias below the threshold voltage is applied to the top electrode and the device is switched to an even higher HRS [Fig. 4(c)]. When a negative bias is applied, the oxygen vacancies can be pulled back and the LRS is obtained [Fig. 4(d)]. In consistence with our experimental data [Fig. 4(e)], within this model, the LRS of the bipolar resistive switching mode is identical with HRS of the unipolar switching mode.

The redox-process responsible for the bipolar switching may also occur at the interface, as generally expected for bipolar switching mode. ${ }^{1,2}$ For unipolar switching, simulations showed that the breakdown of the filament is expected to occur in its middle at the place of the maximum local temperature. ${ }^{19}$ Since unipolar and bipolar switching are cross-linked in our samples by one common resistive branch [Fig. 4(e)], it is likely that the redox-driven bipolar switching occurs also at a considerable distance from the interface.

\section{CONCLUSION}

In conclusion, we have observed the reversible alternation of bipolar and unipolar resistive switching in polycrystalline perovskite BST thin films. When the switching voltage exceeds a certain threshold voltage, the devices exhibit unipolar switching while for switching voltages below the threshold voltage, the devices shows bipolar switching behavior. The LRS of the bipolar switching state is identical with the HRS of the unipolar switching state. We can explain our data consistently with a single filament model, taking into account a reversible voltage-driven redox-process at the trace of the fused filament.

\section{ACKNOWLEDGMENTS}

We thank R. Bruchhaus for critical reading of the manuscript. This work was financially supported by Intel Inc., Santa Clara.

${ }^{1}$ R. Waser and M. Aono, Nature Mater. 6, 833 (2007).

${ }^{2}$ A. Sawa, Mater. Today 11, 28 (2008).

${ }^{3}$ C. B. Lee, B. S. Kang, M. J. Lee, S. E. Ahn, G. Stefanovich, W. X. Xianyu, K. H. Kim, J. H. Hur, H. X. Yin, Y. Park, and I. K. Yoo, Appl. Phys. Lett. 91, 082104 (2007).

${ }^{4}$ A. Chen, S. Haddad, Y. C. Wu, Z. Lan, T. N. Fang, and S. Kaza, Appl. Phys. Lett. 91, 123517 (2007).

${ }^{5}$ C. Y. Lin, C. Y. Wu, T. C. Lee, F. L. Yang, and C. Hu, IEEE Electron Device Lett. 28, 366 (2007).

${ }^{6}$ B. J. Choi, D. S. Jeong, S. K. Kim, C. Rohde, S. Choi, J. H. Oh, H. J. Kim, C. S. Hwang, K. Szot, R. Waser, B. Reichenberg, and S. Tiedke, J. Appl. Phys. 98, 033715 (2005).

${ }^{7}$ S. Q. Liu, N. J. Wu, and A. Ignatiev, Appl. Phys. Lett. 76, 2749 (2000).

${ }^{8}$ A. Beck, J. G. Bednorz, C. Gerber, C. Rossel, and D. Widmer, Appl. Phys. Lett. 77, 139 (2000).

${ }^{9}$ W. Shen, R. Dittmann, U. Breuer, and R. Waser, Appl. Phys. Lett. 93, 222102 (2008).

${ }^{10}$ S. C. Chae, J. S. Lee, S. J. Kim, S. B. Lee, S. H. Chang, C. Liu, B. Kahng, H. Shin, D.-W. Kim, H. Shin, C. U. Jung, S. Seo, M.-J. Lee, and T. W. Noh, Adv. Mater. (Weinheim, Ger.) 20, 1154 (2008).

${ }^{11}$ M.-J. Lee, S. Seo, D.-C. Kim, S.-E. Ahn, D. H. Seo, I.-K. Yoo, I.-G. Baek, D.-S. Kim, I.-S. Byun, S.-H. Kim, I.-R. Hwang, J.-S. Kim, S.-H. Jeon, and B. H. Park, Adv. Mater. (Weinheim, Ger.) 19, 73 (2007).

${ }^{12}$ D. S. Jeong, H. Schroeder, and R. Waser, Electrochem. Solid-State Lett. 10, G51 (2007).

${ }^{13}$ K. Nagashima, T. Yanagida, K. Oka, and T. Kawai, Appl. Phys. Lett. 94, 242902 (2009).

${ }^{14}$ Y. B. Nian, J. Strozier, N. J. Wu, X. Chen, and A. Ignatiev, Phys. Rev. Lett. 98, 146403 (2007).

${ }^{15}$ D. S. Jeong, B. J. Choi, and C. S. Hwang, J. Appl. Phys. 100, 113724 (2006).

${ }^{16}$ R. Waser, R. Dittmann, G. Staikov, and K. Szot, Adv. Mater. (Weinheim, Ger.) 21, 2632 (2009).

${ }^{17}$ C. Park, S. H. Jeon, S. C. Chae, S. Han, B. H. Park, and D.-W. Kim, Appl. Phys. Lett. 93, 042102 (2008).

${ }^{18}$ S. R. Lee, K. Char, D. C. Kim, R. Jung, S. Seo, X. S. Li, G.-S. Park, and I. K. Yoo, Appl. Phys. Lett. 91, 202115 (2007).

${ }^{19}$ D. Ielmini, C. Cagli, and F. Nardi, Appl. Phys. Lett. 94, 063511 (2009). 